\title{
Effects of 2-Octynyladenosine (YT-146) on Mitochondrial Function in Ischemic/Reperfused Rat Hearts
}

\author{
Jun Sasamori, ${ }^{* a}$ Yohei Abe, ${ }^{b}$ Tetsuro Marunouchi, ${ }^{b}$ Yoichi Manome, ${ }^{a}$ Takehiro Uchibori, ${ }^{a}$ and \\ Kouichi Tanonaka ${ }^{b}$ \\ ${ }^{a}$ Drug Research Department, Fukushima Research Laboratories, Toa Eiyo Ltd.; Fukushima 960-0280, Japan: and \\ ${ }^{b}$ Department of Molecular and Cellular Pharmacology, Tokyo University of Pharmacy and Life Sciences; Hachioji \\ 192-0355, Japan.
}

Received August 13, 2015; accepted September 29, 2015

This study investigated the effects of an adenosine receptor agonist, 2-octynyladenosine (YT-146), on mitochondrial function in ischemic and ischemic/reperfused hearts. Isolated rat hearts were perfused in the Langendorff manner with a constant flow rate, and exposed to $30 \mathrm{~min}$ of ischemia followed by $60 \mathrm{~min}$ of reperfusion. Preischemic treatment with YT-146 significantly improved postischemic recovery of left ventricular developed pressure. The high-energy phosphate content in reperfused hearts treated with YT-146 was also more greatly restored than in untreated hearts. YT-146 treatment attenuated the $\mathrm{Na}^{+}$content of a mitochondria-enriched fraction, but not the myocardial $\mathrm{Na}^{+}$content, at the end of ischemia. These results suggest that preischemic YT-146 treatment preserves the energy-producing ability of mitochondria during ischemia in the $\mathrm{Na}^{+}$-accumulated myocardium. YT-146 also attenuated both the sodium lactate-induced decrease in mitochondrial energy-producing ability and the increase in mitochondrial $\mathrm{Na}^{+}$concentration in the myocardial skinned fibers. YT-146 may attenuate $\mathrm{Na}^{+}$influx to myocardial mitochondria in ischemic cardiac cells, resulting in both preservation of the ability of mitochondria to produce energy and enhancement of the contractile recovery in reperfused hearts. Our findings suggest that the cardioprotective effects of YT-146 against ischemia/reperfusion injury are at least partially due to the preservation of mitochondrial function in the ischemic myocardium.

Key words YT-146; adenosine receptor agonist; cardioprotection; mitochondrial function

Many investigators have suggested a close relationship between preserved mitochondrial energy production in ischemic/ reperfused hearts and myocardial contractile recovery during the reperfusion phase. ${ }^{1-3)}$ Mitochondria are the primary intracellular organelles for energy production, which is necessary for both cardiac pump function and cell viability. ${ }^{4,5)}$ Therefore, protection of cardiac mitochondria against ischemia/ reperfusion-induced cellular deterioration may be a critical mechanism underlying cardioprotection in ischemic/reperfused hearts.

Adenosine has multiple biological functions, including cardioprotection against ischemia/reperfusion injury in various animal models. ${ }^{6-8)}$ An adenosine receptor agonist 2-octynyladenosine (YT-146), has been shown to be resistant to adenosine deaminase in vivo. We have recently shown that preischemic treatment with $0.3 \mu \mathrm{M}$ YT-146 may have cardioprotective effects against ischemia/reperfusion injury in isolated rat hearts perfused with a Langendorff mode. 9) In the previous study, we found that YT-146-induced cardioprotective effects against ischemia/reperfusion injury were canceled by treatment of perfused hearts with adenosine $A_{1}$ receptor antagonist and protein kinase $\mathrm{C}$ (PKC) inhibitor, respectively. The results of the study suggested that cardioprotection induced by YT-146 treatment might be due to an agonistic action by the adenosine $\mathrm{A}_{1}$ receptor, followed by PKC activation. ${ }^{9)}$ However, the effects of YT-146 treatment of perfused hearts on mitochondrial energy production in the ischemic myocardium are unknown. In the current study, the effects of YT-146 treatment on mitochondrial function in ischemic and ischemic/reperfused hearts were examined. We also examined the effects of YT-146 on myocardial and mitochondrial abnormal accumulation of $\mathrm{Na}^{+}$content $\left(\mathrm{Na}^{+}\right.$overload), because the effect of adenosine agonist on the relationship between abnormal $\mathrm{Na}^{+}$ accumulation in myocardium and a decrease in mitochondrial function under ischemic conditions is little known.

\section{MATERIALS AND METHODS}

Animals and Agents Male Wistar rats weighing 250-280g (Japan Laboratory Animals Inc., Tokyo, Japan) were used in the current study. The animals were conditioned to an environment at $23 \pm 1^{\circ} \mathrm{C}$ with a constant humidity of $55 \pm 5 \%$ and a 12 -h light/12-h dark cycle and given free access to food and tap water according to the Guide for the Care and Use of Laboratory Animals as promulgated by the National Research Council (National Academy Press, Washington D.C., 1996). The study protocol was approved by the Committee of Animal Care and Welfare of Tokyo University of Pharmacy and Life Sciences.

The following agents were used in this experiment: protease inhibitors (Wako Pure Chemical Industries, Ltd., Osaka, Japan), fatty acid-free bovine serum albumin (BSA) and ethylene glycol tetraacetic acid (EGTA; Sigma Chem. Co., St. Louis, MO, U.S.A.), 2-(N-morpholino)-ethanesulfonate (MOPS) and ethylenediaminetetraacetic acid (EDTA; Dojin Chemical Institute, Kumamoto, Japan), sodium-binding benzofuran isophthalate-acetoxymethyl (SBFI/AM; Molecular Probes, Eugene, OR, U.S.A.), ATP, ADP and creatine phosphate (CP; Roche Diagnostics, Mannheim, Germany).

Perfusion of Isolated Hearts Perfusion of hearts and 
treatment with YT-146 were performed according to previously described methods. ${ }^{10)}$ After diethyl ether anesthesia, the hearts were rapidly isolated, and transferred to the Langendorff apparatus. The hearts were perfused at $37^{\circ} \mathrm{C}$ with a constant flow rate $(9 \mathrm{~mL} / \mathrm{min})$ of Krebs-Henseleit bicarbonate buffer $\left(120 \mathrm{~mm} \mathrm{NaCl}, 4.8 \mathrm{mM} \mathrm{KCl}, 1.2 \mathrm{~mm} \mathrm{KH}_{2} \mathrm{PO}_{4}, 1.2 \mathrm{~mm}\right.$ $\mathrm{MgSO}_{4}, 1.25 \mathrm{~mm} \mathrm{CaCl}_{2}, 25 \mathrm{~mm} \mathrm{NaHCO}_{3}$, and $11 \mathrm{~mm}$ glucose). The perfusion buffer was equilibrated with a gas mixture of $95 \% \mathrm{O}_{2}+5 \% \mathrm{CO}_{2}$ to $\mathrm{pH}$ 7.4. A latex balloon with an uninflated diameter of $3.7 \mathrm{~mm}$ was connected to a pressure transducer (TP-200, Nihonkohden, Tokyo, Japan), and inserted into the left ventricular cavity through the mitral opening. Five $\mathrm{mmHg}$ left ventricular end-diastolic pressure (LVEDP) was initially loaded onto the perfused hearts. Left ventricular developed pressure (LVDP), a convenient marker of cardiac contractile function, was monitored throughout the experiment by a pressure transducer (TP-200) connected to an amplifier (AP-621G, Nihonkohden). Heart rates were measured using a heart rate counter (AT-601G, Nihonkohden). Perfusion pressure of the perfused heart was monitored through a branch of the aortic cannula by means of an another pressure transducer (TP-200). Hemodynamic parameters were recorded on a thermal pen recorder (WT-645G, Nihonkohden). The heart was subjected to 30 -min equilibration and was paced at 300 beats/ min with an electrical stimulator via two directly attached silver electrodes.

Ischemia/Reperfusion and YT-146 Treatment After a 30-min equilibration period, the perfusion was stopped for $30 \mathrm{~min}$ to induce global ischemia as described previously. ${ }^{9)}$ After $30 \mathrm{~min}$ of ischemia, the hearts were reperfused for $60 \mathrm{~min}$ at $37^{\circ} \mathrm{C}$ with the Krebs-Henseleit bicarbonate buffer equilibrated with a gas mixture of $95 \% \mathrm{O}_{2}$ and $5 \% \mathrm{CO}_{2}$. The hearts were paced throughout the experiment except for the first $15 \mathrm{~min}$ of reperfusion to prevent contractile irregularities during this period.

YT-146 was dissolved in the Krebs-Henseleit buffer and infused into the portion of the perfused heart just distal to the aortic cannula for $10 \mathrm{~min}$ prior to the onset of ischemia for a final YT-146 concentration of $0.3 \mu \mathrm{M}$. The hearts were then subjected to ischemia alone or ischemia/reperfusion. ${ }^{9)}$

For purpose of comparison, hearts treated with and without YT-146 treatment were perfused for $90 \mathrm{~min}$ under normoxic conditions (normoxic group).

$\mathrm{Na}^{+}$Content in Myocardium and Mitochondria-Enriched Fraction The $\mathrm{Na}^{+}$content in the myocardium with and without YT-146 pretreatment was measured to assess ionic disturbances in the ischemic heart. At the end of preischemia, ischemia, and reperfusion, hearts were perfused for $1 \mathrm{~min}$ with $8.0 \mathrm{~mL}$ ice-cold $320 \mathrm{~mm}$ sucrose- $20 \mathrm{~mm}$ Tris- $\mathrm{HCl}, \mathrm{pH}$ 7.4. Myocardial $\mathrm{Na}^{+}$was extracted from the dry tissue as described previously. ${ }^{11)}$ The $\mathrm{Na}^{+}$concentration in the extract was measured using an atomic absorption spectrometer (AA-680; Shimadzu, Kyoto, Japan). A previous study confirmed that $\mathrm{Na}^{+}$measured by this method is devoid of contamination from the extracellular and vascular spaces of the heart. ${ }^{11)}$

To determine $\mathrm{Na}^{+}$content in the mitochondria of hearts with and without YT-146 treatment, we prepared mitochondria-enriched fraction from the hearts at the ends of preischemia, ischemia, and reperfusion, respectively. The hearts were minced and then homogenized in ice-cold buffer containing $180 \mathrm{~mm} \mathrm{KCl}, 10 \mathrm{~mm}$ EDTA ( $\mathrm{pH} 7.4)$, and $0.5 \%$ fatty acid-free
BSA (Sigma Chem. Co.). The homogenate was centrifuged at $800 \times \boldsymbol{g}$ for $10 \mathrm{~min}$ at $2^{\circ} \mathrm{C}$, and the resultant supernatant fluid was centrifuged at $8000 \times \boldsymbol{g}$ for $10 \mathrm{~min}$ at $2^{\circ} \mathrm{C}$. After the crude mitochondria were resuspended in the buffer and then centrifuged at $8000 \times \boldsymbol{g}$ for $10 \mathrm{~min}$ at $2^{\circ} \mathrm{C}$, the resultant pellet was resuspended in the suspension buffer $(20 \mathrm{~mm}$ Tris- $\mathrm{HCl}$, $\mathrm{pH} 6.8$, containing $320 \mathrm{~mm}$ sucrose and $0.25 \% \mathrm{BSA}$ ). The $\mathrm{Na}^{+}$ content of the mitochondria-enriched fraction was measured according to the atomic absorption method described above. Protein concentrations of the mitochondria-enriched fraction were determined by the Lowry's method.

Myocardial High-Energy Phosphate After perfusion, the hearts were freeze-clamped with aluminum tongs precooled with liquid nitrogen as described previously to measure myocardial ATP and CP levels. ${ }^{12}$ Briefly, frozen ventricles were pulverized and mixed with $0.3 \mathrm{M} \mathrm{HClO}_{4}$ and $0.25 \mathrm{~mm}$ EDTA under liquid nitrogen cooling. The extract was centrifuged at $8000 \times \boldsymbol{g}$ for $15 \mathrm{~min}$ at $4{ }^{\circ} \mathrm{C}$, and the resulting supernatant sampled to measure myocardial ATP and CP by the HPLC method described previously. ${ }^{12)}$ Myocardial CP was converted to ATP by the creatine kinase enzymatic reaction.

Mitochondrial Oxygen Consumption Rate of Myocardial Skinned Fibers The mitochondrial oxygen consumption rate (OCR), a parameter for mitochondrial activity in the heart, was determined as described previously. ${ }^{13)}$ After perfusion, myocardial fibers 0.3 to $0.4 \mathrm{~mm}$ in diameter and 3 to $4 \mathrm{~mm}$ in length were prepared from the left ventricular free wall using a McIlwain Tissue Chopper (Mickle Lab. Engineering Co., NY, U.S.A.) and transferred to relaxing medium A, composed of $10 \mathrm{~mm}$ EGTA, $3 \mathrm{~mm} \mathrm{MgSO}_{4}, 20 \mathrm{~mm}$ taurine, $0.5 \mathrm{~mm}$ dithiothreitol, $20 \mathrm{~mm}$ imidazole, $160 \mathrm{~mm}$ potassium MOPS, $5 \mathrm{~mm}$ ATP, and $15 \mathrm{~mm} \mathrm{CP} \mathrm{(pH} \mathrm{7.0).} \mathrm{The} \mathrm{myocardial} \mathrm{fibers} \mathrm{were} \mathrm{in-}$ cubated for $20 \mathrm{~min}$ in $1 \mathrm{~mL}$ of medium A containing $75 \mu \mathrm{g} / \mathrm{mL}$ saponin. After incubation, the myocardial fibers were washed for $10 \mathrm{~min}$ in fresh medium B (medium A without ATP and $\mathrm{CP}$, but supplemented with $0.5 \% \mathrm{BSA}$ ) to remove the saponin. All procedures were carried out at $4^{\circ} \mathrm{C}$. The OCR of the skinned fibers was determined using a Clark-type electrode (Central Kagaku, Tokyo, Japan) containing the skinned fibers in $1.0 \mathrm{~mL}$ of medium $\mathrm{B}$ at $30^{\circ} \mathrm{C}$. The basal OCR was measured

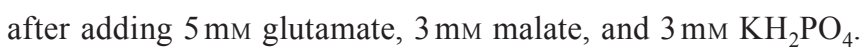
The total (maximal) OCR was measured after further addition of $1 \mathrm{~mm}$ ADP and $7.5 \mathrm{~mm}$ creatine. The ADP-stimulated OCR of the skinned fibers was the difference between the maximal and basal OCRs. After measuring the OCR, the skinned fibers were solubilized with $0.5 \mathrm{~mL}$ of $2 \mathrm{M} \mathrm{NaOH}$ for $30 \mathrm{~min}$ at $60^{\circ} \mathrm{C}$ and the protein concentration measured.

$\mathrm{Na}^{+}$Concentration in Skinned Fibers In another set of experiments, the perfused hearts were removed from the perfusion apparatus after treatment with or without $0.3 \mu \mathrm{M}$ YT-146. The cardiac skinned fibers then were prepared as described above.

The concentration of mitochondrial $\mathrm{Na}^{+}$in the skinned fibers was determined as described by Jung et al., ${ }^{14)}$ with slight modification. A membrane-permeable $\mathrm{Na}^{+}$indicator, SBFI/AM, was dissolved in dimethyl sulfoxide (DMSO) at a concentration of $1 \mathrm{mM}$. Since SBFI/AM is a hydrophobic probe, $3 \mu \mathrm{L}$ of $25 \%(\mathrm{w} / \mathrm{v})$ Pluronic F-127, a nonionic surfactant, was mixed with $3 \mu \mathrm{L}$ of $1 \mathrm{~mm}$ SBFI/AM to enhance mitochondria loading of the hydrophobic sodium indicator in the skinned fibers. The mixture of SBFI/AM and Pluronic 
F-127 was added to $200 \mu \mathrm{L}$ of an incubation buffer composed of $1.5 \mathrm{~mm}$ Tris-ATP ( $\mathrm{pH}$ 7.4). Next, $400 \mu \mathrm{L}$ of skinned fibers suspended in the incubation medium were mixed with $200 \mu \mathrm{L}$ of the incubation medium containing SBFI/AM and incubated for $30 \mathrm{~min}$ at $25^{\circ} \mathrm{C}$. After loading SBFI into mitochondria of the skinned fibers, the suspension was centrifuged at $8000 \times \boldsymbol{g}$ at $25^{\circ} \mathrm{C}$ for $5 \mathrm{~min}$ to remove excess SBFI/AM.

SBFI-loaded mitochondria $(600 \mu \mathrm{L}$ suspension) in $1 \mathrm{~mL}$ cell were placed in a fluorescence analyzer (CAF110, JASCO, Hachioji, Japan), with a 340 and $380 \mathrm{~nm}$ excitation and $500 \mathrm{~nm}$ emission. $\mathrm{Na}^{+}$loaded in the mitochondria due to various concentrations of sodium lactate ranging from 6.25 to $50 \mathrm{~mm}$ was expressed as the ratio of fluorescence emission excited by the two wavelengths. ${ }^{15)}$

Statistics Each value represents the mean \pm standard error of the mean (S.E.M.). Statistical analyses were performed using a statistical package (EXSUS, CAC EXICARE, Osaka, Japan). Statistical significance was evaluated using one-way ANOVA followed by Bonferroni's or Dunnett's multiple comparisons if necessary. Differences with a probability of $5 \%$ or less were considered statistically significant.

\section{RESULTS}

Contractile Function of Perfused Hearts LVDP, LVEDP and perfusion pressure values during ischemia/reperfusion with and without $0.3 \mu \mathrm{M}$ YT-146 treatment are shown in Fig. 1. The baseline (initial) LVDP values for groups with and without YT-146 treatment were $90.0 \pm 3.5 \mathrm{mmHg}$ and $88.0 \pm 2.5 \mathrm{mmHg}$ ( $n=5$ for each group), respectively. The YT-146 concentration $(0.3 \mu \mathrm{M})$ did not affect LVDP values during preischemia. After onset of ischemia, LVDP declined to zero within $3 \mathrm{~min}$, and remained at that value during ischemia. The LVDP recovered to approximately $30 \mathrm{mmHg}$ by the end of reperfusion. In contrast, LVDP was significantly recovered at the end of reperfusion among hearts treated with YT-146.

The LVEDP of the vehicle treated hearts began to rise during ischemia. The LVEDP further increased upon reperfusion, peaking $5 \mathrm{~min}$ after the onset of reperfusion. Although the LVEDP gradually declined during reperfusion, it was approximately $100 \mathrm{mmHg}$ at the end of reperfusion. In contrast, treatment with YT-146 significantly attenuated the rise in LVEDP during ischemia and reperfusion and the LVEDP at the end of reperfusion was approximately $50 \mathrm{mmHg}$.

The perfusion pressure of vehicle treated hearts increased upon reperfusion, peaking $5 \mathrm{~min}$ after the onset of reperfusion. At the end of reperfusion, the perfusion pressure of vehicle treated hearts was approximately $100 \mathrm{mmHg}$. In contrast, treatment with YT-146 significantly attenuated the rise in perfusion pressure during reperfusion and the value at the end of reperfusion was approximately $60 \mathrm{mmHg}$.

Myocardial High-Energy Phosphates Figure 2 shows ATP and CP contents in perfused hearts with and without YT-146 treatment. Myocardial ATP and CP contents at the end of preischemia in the absence of YT-146 were 23.6 \pm 0.5 and $34.0 \pm 1.3 \mu \mathrm{mol} / \mathrm{g}$ dry tissue, respectively $(n=5)$. Their contents
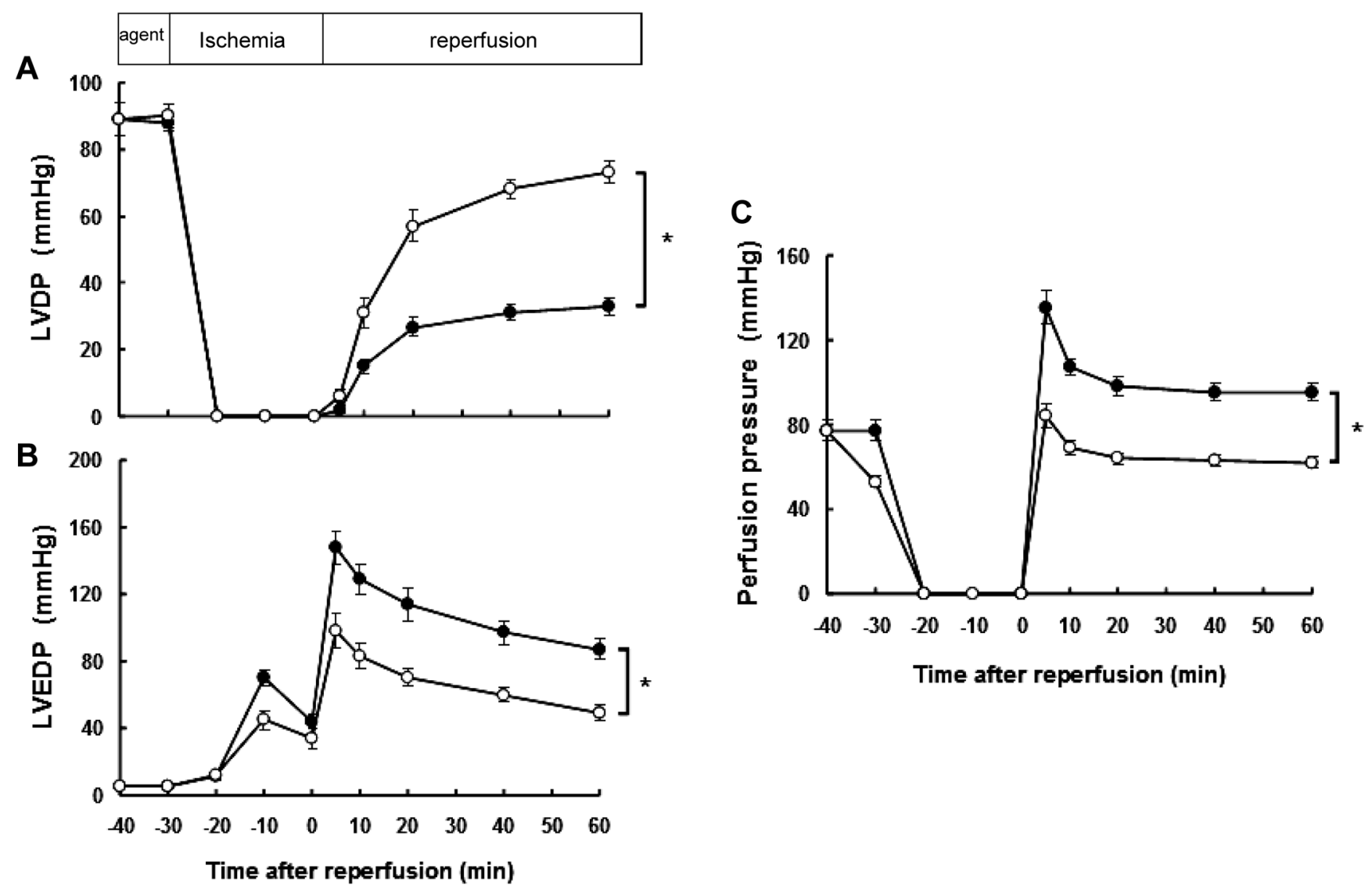

Fig. 1. Time Course of Change in LVDP (A), LVEDP (B) and Perfusion Pressure (C) during the 30-min Ischemia/60-min Reperfusion

Data are shown for hearts treated with (open circles) and without $0.3 \mu \mathrm{M}$ YT-146 (closed circles). Each value represents the mean \pm S.E.M. $(n=5$ ). $*$ Significant difference from the group without YT-146 treatment $(p<0.05)$. 


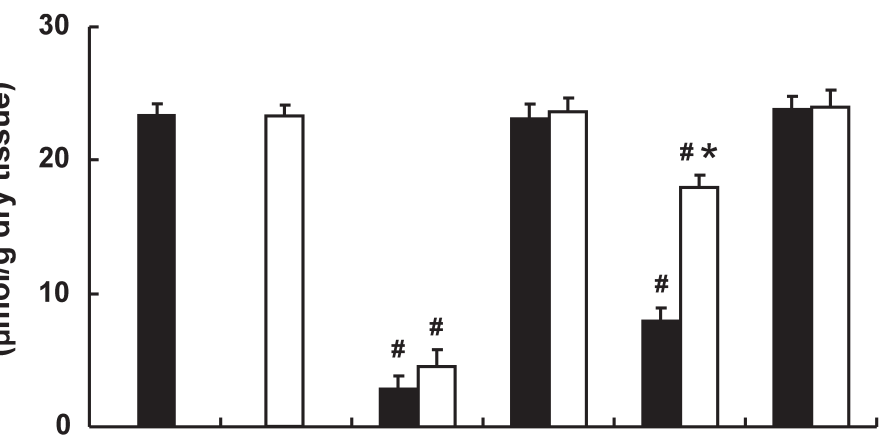

$$
40
$$

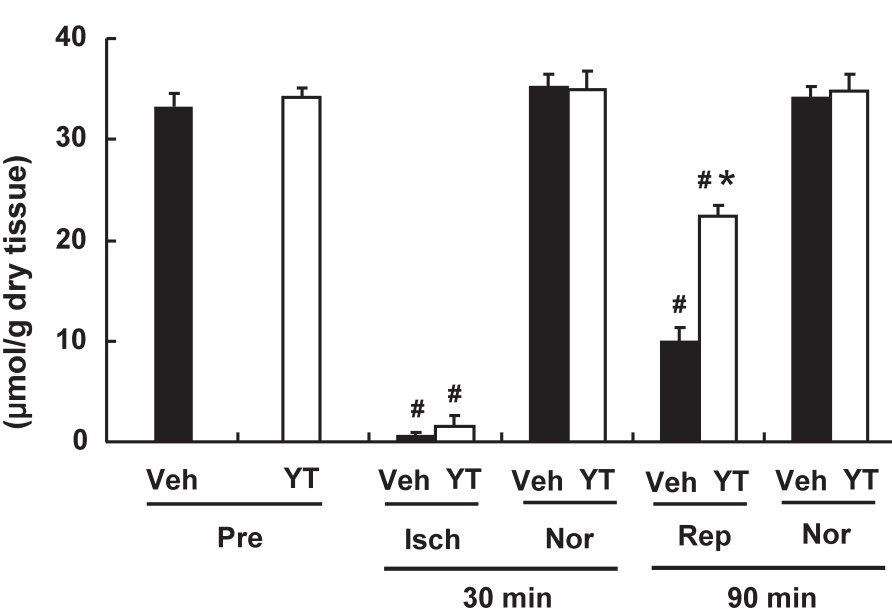

Fig. 2. Myocardial ATP (Upper Panel) and Creatine Phosphate (Lower Panel) Contents at the Ends of Preischemia (Pre), Ischemia (Isch), Reperfusion (Rep), and Normoxic Perfusion (Nor) from Hearts Treated without (Vehicle Treated, Veh; Closed Columns) or with $0.3 \mu \mathrm{M} \mathrm{YT-146} \mathrm{(YT;} \mathrm{Open}$ Columns)

Each value represents the mean \pm S.E.M. $(n=5)$. *Significant difference from the corresponding vehicle treated ischemic or ischemic/reperfused hearts group $(p<0.05)$. \#Significant difference from the corresponding normoxic group $(p<0.05)$.

after $90 \mathrm{~min}$ of normoxia were similar to those at the end of preischemia. Myocardial ATP and CP contents following ischemia were approximately 12 and $2 \%$ of the pre-ischemic values, respectively. After reperfusion, myocardial ATP and $\mathrm{CP}$ contents were restored to approximately 33 and $30 \%$ of the preischemic values, respectively.

Neither myocardial ATP nor CP contents at the ends of preischemia and ischemia were altered by YT-146 treatment. Myocardial ATP and CP contents in the reperfused hearts pretreated with YT-146 were restored at the end of reperfusion (approximately 76 and 66\%, respectively).

Mitochondrial Activity of Perfused Hearts Figure 3 shows the ADP-stimulated mitochondrial OCR at the ends of preischemia, ischemia, and reperfusion, respectively, of skinned fibers prepared from hearts with and without YT-146 treatment. The OCR of preischemic hearts was 52.6 \pm 1.2 nano-atom $\mathrm{O} / \mathrm{min} / \mathrm{mg}$ protein $(n=5)$. The OCR of untreated hearts under ischemic conditions significantly decreased to approximately $45 \%$ of the value in preischemic hearts $(n=5)$. The OCR of the reperfused heart was further decreased to approximately $30 \%$ of the preischemic heart value $(n=5)$. In contrast, treatment with YT-146 preserved the OCR of skinned fibers at the ends of both ischemia and reperfusion (approximately 80 and $85 \%$, respectively; $n=5$ each). There were no significant differences in the OCR of perfused hearts under normoxic conditions with or without YT-146 treatment.

$\mathrm{Na}^{+}$Content in the Myocardium and the MitochondriaEnriched Fraction during Ischemia/Reperfusion Changes in $\mathrm{Na}^{+}$content in myocardium and mitochondria-enriched fraction at the end of preischemia, ischemia and reperfusion are shown in Fig. 4. The baseline myocardial $\mathrm{Na}^{+}$content was $54.57 \pm 0.68 \mu \mathrm{mol} / \mathrm{g}$ dry tissue ( $n=5)$ (upper panel in Fig. 4). The content approximately doubled following ischemia. After reperfusion, the $\mathrm{Na}^{+}$content further increased. At the ends of ischemia and reperfusion, the myocardial $\mathrm{Na}^{+}$content in hearts treated with YT-146 was approximately $90 \mu \mathrm{mol} / \mathrm{g}$ dry tissue and less than $70 \mu \mathrm{mol} / \mathrm{g}$ dry tissue, respectively $(n=6$ each).

The base line of $\mathrm{Na}^{+}$content of the mitochondria-enriched fraction was $35.04 \pm 2.79 \mathrm{nmol} / \mathrm{g} \mathrm{mg}$ protein $(n=4)$ (lower panel in Fig. 4). The $\mathrm{Na}^{+}$content of the mitochondria-enriched fraction in the untreated hearts at the ends of ischemia and reperfusion was increased to approximately 250 and $210 \%$ of the base line value, respectively. In contrast, the $\mathrm{Na}^{+}$content of the corresponding fractions in the YT-146-treated hearts was approximately 180 and $125 \%$ of the base line value, respectively.

There were no changes in $\mathrm{Na}^{+}$content of myocardium and mitochondria-enriched fraction under normoxic conditions regardless of YT-146 treatment.

Effect of YT-146 on $\mathrm{Na}^{+}$Entry into Mitochondria in Skinned Fibers Figure 5 shows changes in SBFI signal of mitochondria within skinned fibers. When skinned fibers were incubated with various concentrations (ranging from 6.25 to $50 \mathrm{~mm}$ ) of sodium lactate, a possible glycolytic metabolite in ischemic hearts, when the skinned fibers were incubated, the SBFI signal increase in the presence of sodium lactate was concentration-dependent. In contrast, the sodium lactate-in- 


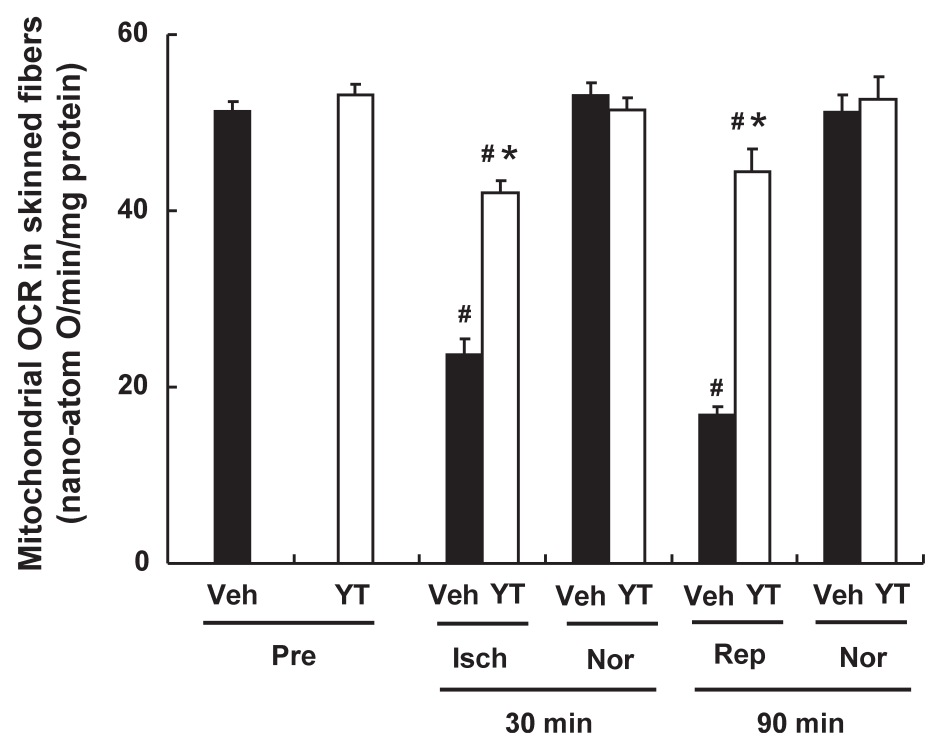

Fig. 3. Mitochondrial Oxygen Consumption Rate (OCR) of Left Ventricular Skinned Fibers Prepared Following Presichemia (Pre), Ischemia (Isch), Reperfusion (Rep), and Normoxic Perfusion (Nor) from Hearts without (Vehicle Treated, Veh; Closed Columns) or with $0.3 \mu \mathrm{M}$ YT-146 Treatment (YT; Open Columns)

Each value represents the mean \pm S.E.M. $(n=5)$. ${ }^{*}$ Significant difference from the corresponding vehicle treated ischemic or ischemic/reperfused hearts group $(p<0.05)$. \#Significant difference from the corresponding normoxic group $(p<0.05)$.
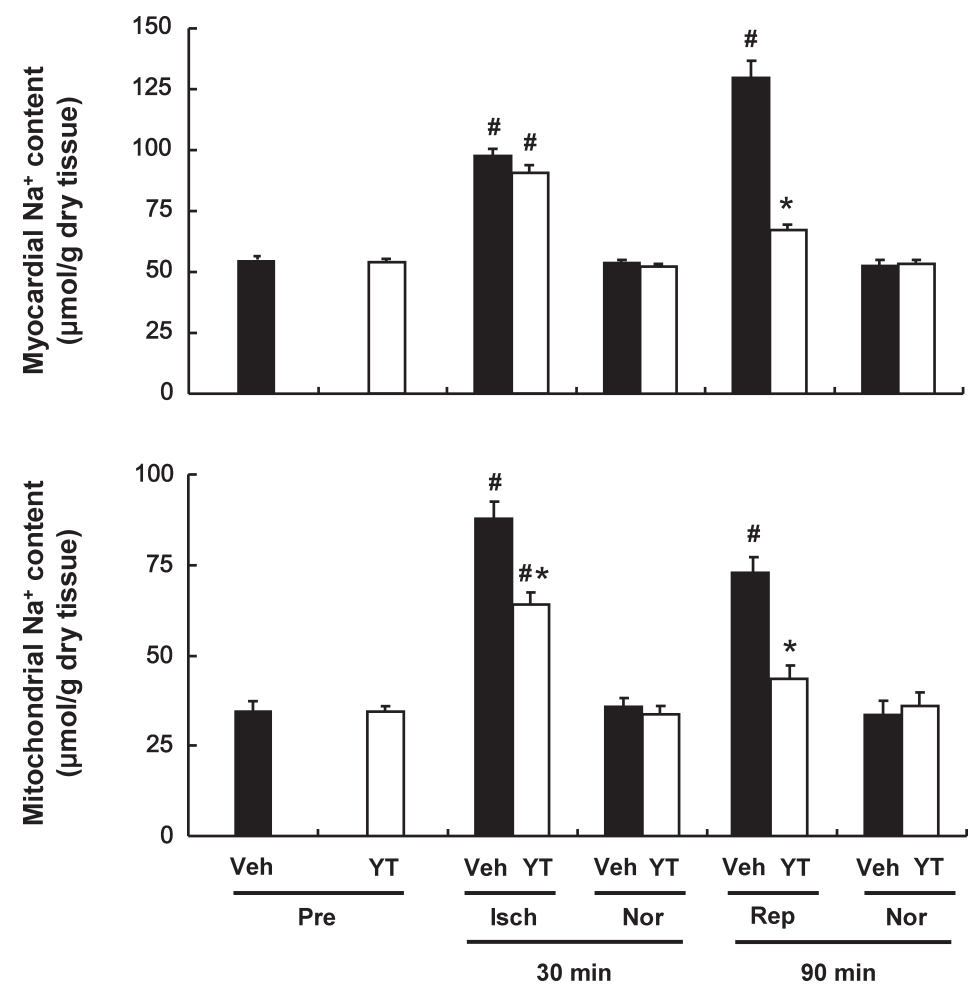

Fig. 4. Myocardial (Upper Panel) and Mitochondrial (Lower Panel) $\mathrm{Na}^{+}$Content at the Ends of Preischemia (Pre), Ischemia (Isch), Reperfusion (Rep), and Normoxic Perfusion (Nor) from Hearts without (Vehicle Treated, Veh; Closed Columns) or Treated with 0.3 $\mu \mathrm{m}$ YT-146 (YT; Open Columns)

Each value represents the mean \pm S.E.M. $(n=5)$. *Significant difference from the corresponding vehicle treated ischemic or ischemic/reperfused hearts group $(p<0.05)$. ${ }^{\#}$ Significantly different from the corresponding normoxic group $(p<0.05)$.

duced increase in SBFI signal was attenuated in skinned fibers prepared from hearts treated with YT-146.

To examine the osmotic effect of sodium salts on SBFI, skinned fibers were incubated in various concentrations of choline chloride. Choline chloride did not affect the SBFI signal, suggesting that any contribution of sodium salts to the osmotic pressure of the SBFI fluorescence ratio is unlikely.

Effect of YT-146 on Mitochondrial Respiration in the Presence of $\mathrm{Na}^{+}$(Sodium Lactate) To examine the effects of $\mathrm{Na}^{+}$on the ability of mitochondria to generate ATP, the mitochondrial OCR of skinned fibers prepared from normal hearts was measured in vitro in the presence and absence of 
sodium lactate (Fig. 6). When skinned fibers were incubated with sodium lactate ranging from 6.25 to $50 \mathrm{~mm}$, the OCR decreased in a concentration-dependent manner.

Next, the OCR of skinned fibers prepared from YT146-treated hearts in the presence of sodium lactate was determined. YT-146 treatment of perfused hearts in the presence of sodium lactate resulted in attenuation of the decrease in the OCR of skinned fibers.

\section{DISCUSSION}

Since the affinity value of adenosine for adenosine $A_{1}$

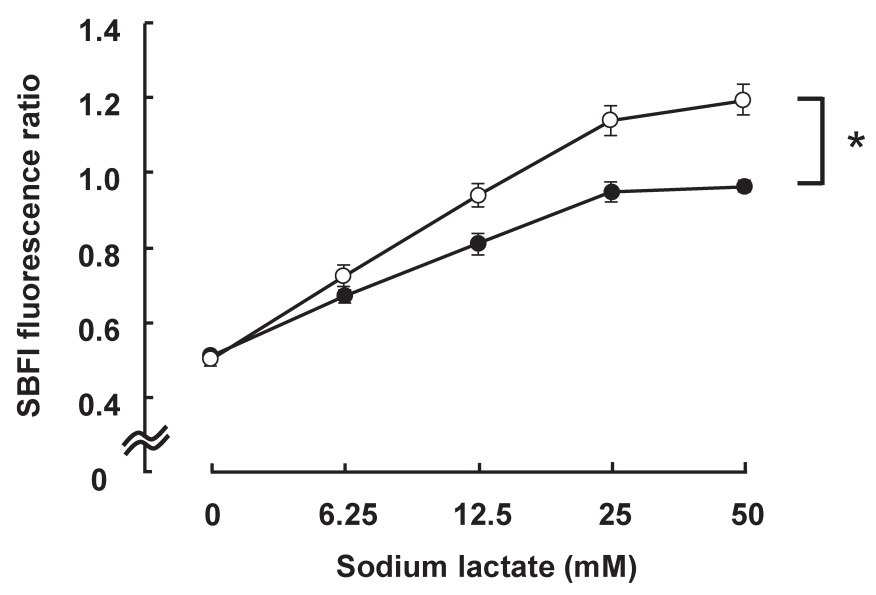

Fig. 5. Sodium Lactate-Induced Increase in Fluorescence Ratio of Sodium-Binding Benzofuran Isophthalate (SBFI)-Loaded Skinned Fibers

Skinned fibers prepared from perfused hearts treated with (open circles) and without $0.3 \mu \mathrm{M}$ YT-146 (closed circles) were incubated with SBFI-AM, a membrane-permeable $\mathrm{Na}^{+}$indicator. After removing unloaded indicator into cardiac mitochondria, the skinned fibers were incubated in 6.25 to $50 \mathrm{~mm}$ sodium lactate. Each value represents the mean \pm S.E.M. of 5 experiments. *Significant difference from the group without YT-146 treatment $(p<0.05)$. receptors is similar to that for adenosine $A_{2}$ receptors, the affinity ratio $\left(A_{1} / A_{2}\right)$ of adenosine for adenosine $A_{1}$ and $A_{2}$ receptors is approximately $1 .{ }^{16)}$ On the other hand, YT-146 exerted approximately 6-fold higher affinity for adenosine $\mathrm{A}_{2 \mathrm{~A}}$ receptors than that for adenosine $\mathrm{A}_{1}$ receptors. ${ }^{17)}$ Kogi et al. reported that an agonistic activity of YT-146 for adenosine $\mathrm{A}_{1}$ receptor was similar to that of adenosine. ${ }^{18)}$ We previously reported that YT-146 increased acute hyperemic coronary flow immediately after reperfusion in in vivo canine ischemic/ reperfused model. ${ }^{19)}$ When effects of YT-146 on cardiac contractile function during ischemia/reperfusion in the previous study were examined, we found that YT-146 exerts cardioprotective effects via stimulation of the adenosine $\mathrm{A}_{1}$ receptor in isolated, perfused rat hearts. ${ }^{9)}$ We have also found that optimal concentration of YT-146 for cardioprotection against ischemia/ reperfusion injury in isolated, perfused rat hearts is $0.3 \mu \mathrm{M}$. However, when the isolated hearts were perfused using the constant perfusion pressure mode of the Langendorff method, $0.3 \mu \mathrm{M}$ YT-146 treatment resulted in markedly increased coronary flow rate due to adenosine $\mathrm{A}_{2}$ receptor-mediated coronary artery dilation. ${ }^{19,20)}$ In this study, we therefore employed a perfusion method with a constant flow rate. We also found that YT-146 treatment of perfused hearts enhanced the postischemic recovery of cardiac contractile function, whereas the supply of perfusate was constant during preischemia and reprfusion periods. Our findings in this study suggest that the effect of YT-146-induced increase in coronary flow on cardioprotection against ischemia/reperfusion injury is smaller under present experimental conditions.

In this study, YT-146 treatment resulted in an enhanced restoration of myocardial high-energy phosphate content such as ATP and CP in reperfused hearts. We also found that YT-146 treatment preserved the ability of mitochondria to produce energy in the heart under ischemic conditions by using skinned fibers. These findings suggest that YT-146 treatment preserves

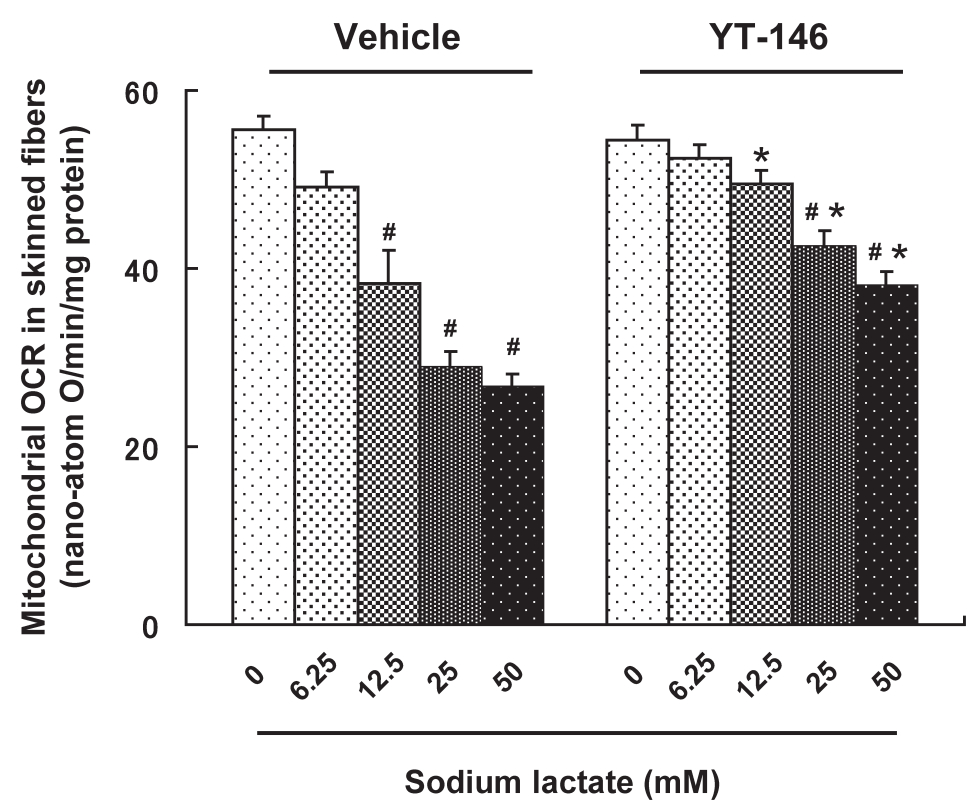

Fig. 6. Sodium Lactate-Induced Decrease in the Mitochondrial Oxygen Consumption Rate (OCR) of Skinned Fibers Prepared from the Left Ventricular Muscles

Skinned fibers were prepared from perfused hearts treated without (left-side chart; vehicle) and with $0.3 \mu \mathrm{M}$ YT-146 (right-side chart; YT-146) and incubated in 6.25 to $50 \mathrm{~mm}$ sodium lactate. Each value represents the mean \pm S.E.M. $(n=5)$. ${ }^{*}$ Significant difference from the corresponding vehicle treated hearts group $(p<0.05)$. ${ }^{\#}$ Significant difference from the corresponding normoxic group $(p<0.05)$. 
mitochondrial function in the ischemic/reperfused hearts.

In previous studies, we have shown that $\mathrm{Na}^{+}$overload in the ischemic myocardium induces an irreversible decrease in the ability of mitochondria to produce energy, leading to contractile failure of the reperfused heart. ${ }^{21)} \mathrm{Na}^{+}$overload is induced by an increased $\mathrm{Na}^{+}$flux form extracellular space to intracellular space via $\mathrm{Na}^{+}$channels and $\mathrm{Na}^{+} / \mathrm{H}^{+}$exchangers. ${ }^{22,23)}$ However, measurement of the myocardial $\mathrm{Na}^{+}$content at the end of ischemia in the present study revealed that the cation content of hearts treated with YT-146 was slightly, but not significantly, lower than that of the untreated hearts, suggesting that YT-146 does not affect these $\mathrm{Na}^{+}$influx pathway. Therefore, we determined changes in the $\mathrm{Na}^{+}$content of mitochondria-enriched fraction in the hearts treated with and without YT-146 at the ends of ischemia and reperfusion. We found that the $\mathrm{Na}^{+}$content of the mitochondria-enriched fraction prepared from the heart treated with YT-146 at the end of ischemia was smaller than that without agent, suggesting that YT-146 treatment attenuates ischemia-induced increase in $\mathrm{Na}^{+}$ content of the mitochondria-enriched fraction, that is, mitochondrial $\mathrm{Na}^{+}$overload. Taken together, our findings suggest that YT-146 treatment preserves the energy-producing ability in cardiac cells under ischemic conditions via an attenuation of mitochondrial $\mathrm{Na}^{+}$overload.

We further examined the effects of YT-146 on $\mathrm{Na}^{+}$overload-induced changes in the ability of mitochondria to produce energy to explore its possible cardioprotective actions. Cardiac skinned fibers were prepared from YT-146-treated hearts, which were not exposed to ischemia/reperfusion. Cardiac skinned fibers were incubated with various concentrations of sodium lactate, a possible metabolites accumulated in ischemic myocardium. The incubation of skinned fibers with sodium lactate increased the SBFI fluorescence ratio, an indicator for $\mathrm{Na}^{+}$concentration, suggesting that $\mathrm{Na}^{+}$overload results in increased $\mathrm{Na}^{+}$flux into mitochondria, which led to decreased mitochondrial energy production. We showed in a previous study that SBFI fluorescence from isolated mitochondria prepared from rat hearts increased in the presence of sodium lactate. ${ }^{21)}$ Our findings using skinned fibers in this study were similar to those in the previous study using isolated mitochondria. Therefore, the increased fluorescent signal from the skinned fibers loaded with SBFI may be due to $\mathrm{Na}^{+}$ accumulation in the cardiac mitochondria due to the presence of sodium lactate.

Furthermore, the mitochondrial OCR was measured in the cardiac skinned fibers incubated in various concentrations of sodium lactate. ${ }^{22,24)}$ The OCR reduction in the presence of sodium lactate was concentration-dependent. The degree of OCR reduction in the presence of sodium lactate was reduced in the skinned fibers prepared from YT-146 treated hearts. YT-146 treatment in vitro attenuated both sodium lactate-induced decrease in mitochondrial energy-producing ability and increase in mitochondrial $\mathrm{Na}^{+}$concentration in the skinned fibers. Therefore, YT-146 may attenuate $\mathrm{Na}^{+}$influx into mitochondria in ischemic cardiac cells with $\mathrm{Na}^{+}$overload, thus preserving mitochondrial energy-producing ability in the ischemic hearts and then enhanced contractile recovery in reperfused hearts.

In summary, postischemic recovery of contractile function of perfused rat hearts was enhanced by YT-146 treatment. YT-146 treatment reduced mitochondrial $\mathrm{Na}^{+}$overload and preserved energy-producing ability of mitochondria in the ischemic myocardium, whereas myocardial $\mathrm{Na}^{+}$accumulation was not attenuated. In in vitro experiments using cardiac skinned fibers, YT-146 treatment attenuated $\mathrm{Na}^{+}$accumulation in mitochondria and also preserved mitochondrial ability for energy production in the presence of sodium lactate. Our findings suggest that YT-146 exerts cardioprotective effects via preservation of mitochondrial function in the ischemic myocardium with $\mathrm{Na}^{+}$overload, leading to an enhancement of postischemic contractile function with a restoration of myocardial high-energy phosphates in the perfused rat hearts.

Conflict of Interest Jun Sasamori, Yoichi Manome and Takehiro Uchibori are employees of Toa Eiyo Ltd.

\section{REFERENCES}

1) Ferrari R. The role of mitochondria in ischemic heart disease. $J$. Cardiovasc. Pharmacol., 28 (Suppl. 1), S1-S10 (1996).

2) Nohl H, Koltover V, Stolze K, Esterbauer H. Ischemia/reperfusion impairs mitochondrial energy conservation and triggers $\mathrm{O}_{2}{ }^{-}$release as a byproduct of respiration. Free Radic. Res. Commun., 18, 127-137 (1993).

3) Takeo S, Tanonaka K, Iwai T, Motegi K, Hirota Y. Preservation of mitochondrial function during ischemia as a possible mechanism for cardioprotection of diltiazem against ischemia/reperfusion injury. Biochem. Pharmacol., 67, 565-574 (2004).

4) Andres J, Flameng W, van Belle H. Energetic state of the postischemic myocardium and its relation to contractile failure. J. Physiol. Pharmacol., 45, 91-103 (1994).

5) Regitz V, Paulson DJ, Hodach RJ, Little SE, Schaper W, Shug AL. Mitochondrial damage during myocardial ischemia. Basic Res. Cardiol., 79, 207-217 (1984).

6) Ely SW, Mentzer RM Jr, Lasley RD, Lee BK, Berne RM. Functional and metabolic evidence of enhanced myocardial tolerance to ischemia and reperfusion with adenosine. J. Thorac. Cardiovasc. Surg., 90, 549-556 (1985).

7) Liu GS, Thornton J, Van Winkle DM, Stanley AW, Olsson RA, Downey JM. Protection against infarction afforded by preconditioning is mediated by $\mathrm{A}_{1}$ adenosine receptors in rabbit heart. Circulation, 84, 350-356 (1991).

8) Olafsson B, Forman MB, Puett DW, Pou A, Cates CU, Friesinger $\mathrm{GC}$, Virmani R. Reduction of reperfusion injury in the canine preparation by intracoronary adenosine: importance of the endothelium and the no-reflow phenomenon. Circulation, 76, 1135-1145 (1987).

9) Sasamori J, Aihara K, Uchibori T, Takahashi A, Takeo S, Tanonaka K. Cardioprotective effects of 2-octynyladenosine (YT-146) in ischemic/reperfused rat hearts. J. Cardiovasc. Pharmacol., 57, 166-173 (2011).

10) Tanonaka K, Iwai T, Motegi $\mathrm{K}$, Takeo S. Effects of $N$-(2-mercaptopropionyl)-glycine on mitochondrial function in ischemic-reperfused heart. Cardiovasc. Res., 57, 416-425 (2003).

11) Tanonaka K, Kajiwara H, Kameda H, Takasaki A, Takeo S. Relationship between myocardial cation content and injury in reperfused rat hearts treated with cation channel blockers. Eur. J. Pharmacol., 372, 37-48 (1999).

12) Iwai T, Tanonaka K, Koshimizu M, Takeo S. Preservation of mitochondrial function by diazoxide during sustained ischaemia in the rat heart. Br. J. Pharmacol., 129, 1219-1227 (2000).

13) Sanbe A, Tanonaka K, Hanaoka Y, Katoh T, Takeo S. Regional energy metabolism of failing hearts following myocardial infarction. J. Mol. Cell. Cardiol., 25, 995-1013 (1993).

14) Jung DW, Apel LM, Brierley GP. Transmembrane gradients of free $\mathrm{Na}^{+}$in isolated heart mitochondria estimated using a fluorescent probe. Am. J. Physiol., 262, C1047-C1055 (1992). 
15) Donoso P, Mill JG, O’Neill SC, Eisner DA. Fluorescence measurements of cytoplasmic and mitochondrial sodium concentration in rat ventricular myocytes. J. Physiol., 448, 493-509 (1992).

16) Abiru T, Yamaguchi T, Watanabe Y, Kogi K, Aihara K, Matsuda A. The antihypertensive effect of 2-alkynyladenosines and their selective affinity for adenosine A2 receptors. Eur. J. Pharmacol., 196, 69-76 (1991).

17) Konno T, Murakami A, Uchibori T, Nagai A, Kogi K, Nakahata N. Involvement of adenosine $\mathrm{A}_{2 \mathrm{a}}$ receptor in intraocular pressure decrease induced by 2-(1-octyn-1-yl)adenosine or 2-(6-cyano-1-hexyn1-yl)adenosine. J. Pharmacol. Sci., 97, 501-509 (2005).

18) Kogi K, Uchibori T, Aihara K, Yamaguchi T, Abiru T. Pharmacological profile of the 2-alkynyladenosine derivative 2-octynyladenosine (YT-146) in the cardiovascular system. Jpn. J. Pharmacol., 57, 153-165 (1991).

19) Sasamori J, Aihara K, Yoneyama F, Sato I, Kogi K, Takeo S. Amelioration of ischemia/reperfusion-induced myocardial infarction by the 2-alkynyladenosine derivative 2-octynyladenosine (YT-146). J. Cardiovasc. Pharmacol., 47, 614-620 (2006).
20) Yoneyama F, Aihara K, Kogi K, Satoh K, Taira N. Similarity and dissimilarity in mode and mechanism of action between YT-146, a selective adenosine receptor $\mathrm{A}_{2}$ agonist, and adenosine in isolated canine hearts. Tohoku J. Exp. Med., 188, 31-45 (1999).

21) Tanonaka K, Motegi K, Arino T, Marunouchi T, Takagi N, Takeo S. Possible pathway of $\mathrm{Na}^{(+)}$flux into mitochondria in ischemic heart. Biol. Pharm. Bull., 35, 1661-1668 (2012).

22) Iwai $T$, Tanonaka $K$, Inoue $R$, Kasahara $S$, Kamo N, Takeo S. Mitochondrial damage during ischemia determines post-ischemic contractile dysfunction in perfused rat heart. J. Mol. Cell. Cardiol., 34, 725-738 (2002)

23) Motegi K, Tanonaka K, Takenaga Y, Takagi N, Takeo S. Preservation of mitochondrial function may contribute to cardioprotective effects of $\mathrm{Na}^{+} / \mathrm{Ca}^{2+}$ exchanger inhibitors in ischaemic/reperfused rat hearts. Br. J. Pharmacol., 151, 963-978 (2007).

24) Iwai $T$, Tanonaka $K$, Inoue $R$, Kasahara $S$, Motegi $K$, Nagaya $S$, Takeo S. Sodium accumulation during ischemia induces mitochondrial damage in perfused rat hearts. Cardiovasc. Res., 55, 141-149 (2002). 